\title{
Arquitectura moderna: puente para la dignidad de la vivienda popular en Colombia ${ }^{1}$
}

\author{
Modern architecture: bridge for the dignity of popular \\ housing in Colombia
}

Cómo citar:

Toro Ospina, A. \& Alami Zambrano, A. M. 2020. Arquitectura moderna: puente para la dignidad de la vivienda popular en Colombia. Designia, 8(1), pp.143-159.

\section{${ }^{1}$ El presente artículo es un avances de la alternativa de grado en investigación como aporte al semillero de APQUA del grupo de investigación PAME de la Universidad de Boyacá .}

* Arquitecta de la Universidad de Los Andes, Master of Science en Conservación de patrimonio y desarrollo urbano de la Technische Universität Dresden Alemania, doctorado en curso en la Bauhaus Universität Weimar, docente de la Universidad de Boyacá, integrante del grupo de investigación PAME. E-mail: atoro@uniboyaca.edu.co, atoro1111@yahoo.es ORCID: https://orcid.org/0000-0001-6493-2665

** Estudiante de pregrado en Arquitectura de la Universidad de Boyacá, integrante del Semillero Apqua. E-mail: alamiana@outlook.com ORCID: https://orcid.org/0000-0002-5519-4143
Palabras clave:

Arquitectura moderna, vivienda popular, vivienda digna

Key words:

Modern architecture, popular housing, worthy housing

Recibido: 29/04/2020

Aceptado: 22/05/2020

\section{Resumen:}

El presente artículo es resultado de la investigación adelantada como alternativa de grado como aporte al semillero de APQUA del grupo de investigación PAME de la Universidad de Boyacá.

La arquitectura moderna tuvo una valiosa participación dentro del proceso de dignificación de la vivienda popular en Colombia. A raíz de la industrialización del país entre las décadas de 1830 y 1850 (Mayor Mora, 2002), el problema de escasez y baja calidad de la vivienda para el sector 
popular se intensificó debido a la migración de campesinos a la ciudad en busca de oportunidades laborales y a la alta demanda de mano de obra. Hasta antes de 1918, el Estado colombiano no prestó la atención requerida a esta situación; las condiciones precarias de las viviendas y la carencia de higiene afectaban directamente a las familias y a la ciudad

En el transcurso de la investigación, al indagar sobre la arquitectura moderna en Colombia y sobre el desarrollo y evolución de la vivienda popular, se encontró que, inicialmente, fueron las comunidades religiosas católicas y las entidades de beneficencia las que se preocuparon ante el desalentador panorama, por lo que comenzaron las construcción de viviendas con un esquema social participativo, de manera conjunta con los beneficiados, con diseños que respondían a los parámetros básicos de la arquitectura moderna en cuanto a iluminación, ventilación, amplitud y flexibilidad de los espacios; estas fueron las primeras viviendas dignas para clases populares en el país.

A inicios de la década de 1920, el Gobierno expidió leyes tendientes a promover la construcción de vivienda popular bajo lineamientos que aplicaban los parámetros básicos modernos, los cuales garantizaban el bienestar de las familias de ingresos bajos. Estos nuevos proyectos de vivienda fueron diseñados por arquitectos colombianos llegados al país desde Europa después de terminar allí sus estudios, influenciados por las nuevas tendencias de la arquitectura moderna. Fue un momento en el que el arquitecto comenzó a tener un rol fundamental en el desarrollo de las ciudades colombianas.

La aplicación de los conceptos de la arquitectura moderna en construcciones populares dio como resultado espacios higiénicos, confortables, flexibles y progresivos, brindando una vivienda digna a la clase obrera, víctima hasta entonces del olvido del Estado, que por mucho tiempo pasó por alto sus necesidades y derechos. La vivienda popular, construida con materiales económicos y aplicando 
los principios de iluminación, ventilación y amplitud de espacios, dio nueva esperanza y mejoró las condiciones de una clase social afectada por el déficit de vivienda; esto da un giro de $180^{\circ}$ a la situación, pues a partir de este momento, la arquitectura moderna ya no es exclusivamente para clases altas, sino que se convierte en una solución para diversas problemáticas de las clases media y baja.

A finales de la década de 1960, el Estado empezó a involucrar compañías inmobiliarias en estos proyectos de vivienda popular, al resultarle costoso su diseño por parte de arquitectos reconocidos, que cobraban altas sumas de dinero por su trabajo. Adicionalmente, los presupuestos para estas viviendas disminuyeron, lo cual desalentó a muchos arquitectos que querían generar calidad, pero para ello se requería de una inversión justa. En conclusión, aquella calidad de las viviendas populares que se había alcanzado gracias a la aplicación de los parámetros de la arquitectura moderna durante la primera mitad del siglo XX, comenzó a disminuir progresivamente, perdiéndose poco a poco los logros alcanzados.

Lo que ocurrió en años posteriores fue el camino a la crítica situación actual que presenta la vivienda de interés social. A partir de la creación del Sistema Nacional de Vivienda de Interés Social en 1991, integrado por entidades públicas y privadas, se estableció el subsidio familiar de vivienda, lo que significó un cambio sustancial en el papel del Estado en la solución de la problemática de vivienda popular, pues pasó de ser promotor, a ser simplemente un facilitador del mercado.

La investigación contempla una comparación entre la vivienda popular influida por la arquitectura moderna y la vivienda de interés social de hoy. Actualmente, las normativas sobre vivienda de interés social se centran en la exigencia de dimensiones mínimas de los espacios con una destinación definida; dado que la norma no contempla aspectos fundamentales de una vivienda digna como la espacialidad y las áreas libres interiores, la iluminación y ventilación, el tratamiento paisajístico en las urbanizaciones, los equipamientos comunales, el acceso a transporte público, entre otros, estos aspectos no son ya tenidos en cuenta en los diseños. Lo que debería ser un derecho, ahora es un privilegio que, lamentablemente, la mayoría de las personas "beneficiadas" con Viviendas de Interés Social, no tienen. 


\section{Abstract:}

This article is the result of the research advanced as a undergraduate alternative as a contribution to the APQUA hotbed of the PAME research group of the University of Boyacá.

Modern architecture had a valuable participation in the process of dignification of popular housing in Colombia. Following the industrialization process that began in Colombia between the 1830s and 1850s (Major Mora, 2002), the problem of shortage and low housing quality for the popular sector intensified due to the migration of peasants to the city in search of job opportunities and high demand for labor. The Colombian State, before 1918, did not pay the required attention to this situation; precarious housing conditions and lack of hygiene directly affected families and the city.

In the course of the research, in research into modern architecture in Colombia and the development and evolution of popular housing, it was found that, initially, it was Catholic religious communities and charities that cared about such a daunting landscape and began housing construction with a participatory social scheme, together with the beneficiaries, with designs that responded to the basic parameters of modern architecture in terms of lighting, ventilation, spaciousness and flexibility of spaces; these were the first decent homes for popular classes in the country.

In the early 1920s, the government issued laws aimed at promoting the construction of popular housing under guidelines that applied modern basic parameters, which guaranteed the well-being of low-income families. These new housing projects were designed by Colombian architects who came to the country from Europe after finishing their studies there, influenced by the new trends of modern architecture. It was a time when the architect began to play a fundamental role in the development of Colombian cities.

The application of the concepts of modern architecture in popular buildings configured hygienic, comfortable, flexible and progressive spaces, providing a dignified home to the working class, a victim until then of the forgetfulness of the state, which for a long time ignored their needs and rights. The popular dwelling, built with economic materials and applying the principles of lighting, ventilation and spaciousness of spaces, gave new hope and improved the conditions of a social class affected by the housing deficit; this takes a 180-degree 
turn to the situation, because from this moment on, modern architecture is no longer exclusively for the upper classes, but becomes a solution for various problems of the middle and lower classes.

In the late 1960s, the state began to involve real estate companies in these popular housing projects, as it was expensive to design by renowned architects, who charged high sums of money for their work. In addition, budgets for these homes decreased, which discouraged many architects who wanted to generate quality, but for this it required a fair investment. In conclusion, that quality of popular housing that had been achieved thanks to the application of the parameters of modern architecture during the first half of the twentieth century, began to decrease progressively, gradually losing the achievements achieved.

What happened in later years was the path to the current critical situation presented by housing of social interest. Since the creation of the National Housing System of Social Interest in 1991, composed of public and private entities, the family housing allowance was established, which meant a substantial change in the role of the State in solving the popular housing problem, since it went from being a promoter, to simply a market facilitator.

The research envisages a comparison between popular housing influenced by modern architecture and today's social housing. Currently, housing regulations of social interest focus on the requirement of minimum dimensions of spaces with a defined destination; fundamental aspects of decent housing such as spatiality and indoor free areas, lighting and ventilation, landscape treatment in developments, communal facilities, access to public transport, among others, are no longer taken into account. What should be a right is now a privilege that, unfortunately, most people "benefited" with Social Interest Housing do not have. 


\section{INTRODUCCIÓN}

La arquitectura moderna en Latinoamérica aplicada a la vivienda popular influyó en gran manera en el desarrollo de Colombia, pues fue uno de los grandes resultados de la industrialización, hecho trascendente en la época moderna. La industrialización hizo más productivos a los ciudadanos, y por ende, más conscientes de sus necesidades y derechos por el simple hecho de ser trabajadores contribuyentes a esta nueva era moderna y al avance acelerado que esta generaba en el país.

La arquitectura moderna popular se convirtió en el puente de integración de la sociedad latina con el mundo cosmopolita. Antes de su llegada, para la clase obrera era normal vivir en condiciones reducidas y antihigiénicas que daban como consecuencia enfermedades de toda índole y constantes conflictos sociales. Las personas no vislumbraban una pronta solución de sus necesidades.

Son pocos los estudios desde la historia de la arquitectura en Colombia que arrojan conclusiones sobre la importancia de la llegada de la arquitectura moderna al país; de igual manera, no han investigado sobre la involución de la vivienda para clases populares, representada hoy en la Vivienda de Interés Social. Por tal motivo, la presente investigación busca mostrar cómo los proyectos de vivienda popular en Colombia entre los años 1930 a 1960, aplicando los conceptos de la arquitectura moderna, respondieron a las necesidades de habitabilidad y de calidad de vida de la sociedad del momento, lo que no ocurre con la vivienda de interés social actual.

\section{Evolución de la vivienda popular en Colombia. El desalentador panorama de la vivienda obrera a comienzos del siglo $\mathrm{XX}$}

A finales del siglo XIX y principios del XX, la clase obrera en Colombia, vivía en espacios reducidos, pobres, obscuros y sucios (Cotrino Diaz, 2007, pág. 7). Entre 1918 y 1945 un considerable número de habitantes de sectores rurales y urbanos vivían en condiciones de hacinamiento y falta de servicios públicos (Arango, 1993, pág. 204), en espacios insalubres que producían lástima y asco.

El sector obrero fue fundamental para el proceso de industrialización del país por lo que la necesidad de generar un lugar digno donde la clase popular pudiera vivir, supliendo sus necesidades básicas, garantizando su comodidad y seguridad con una integración de la vivienda y la industria, debería resolverse de manera urgente para solucionar la desalentadora situación en la que vivían obreros que 
eran explotados por sus patrones, dadas las malas condiciones laborales sumadas a las condiciones precarias de sus viviendas. Si bien, algunos corrieron con la suerte de ser beneficiados por comunidades religiosas y organizaciones benéficas que les brindaron soluciones de vivienda, la mayoría continuó padeciendo esa problemática por varios años. Estas soluciones de vivienda que surgieron a finales de la década de 1910 amortiguaron la dura situación, pues el vivir ahora en un lugar digno les permitió ver la vida con esperanza.

Preocupado porque el descontento de la clase obrera a causa de las malas condiciones de la vivienda llevara a revoluciones sociales y comunistas, el Estado comenzó a hacer frente a la situación. (Cotrino Diaz, 2007, pág. 6). En 1918, la Junta Central de Higiene de Bogotá expidió el Acuerdo 40 sobre higiene en construcciones y sobre el proceso de urbanización de la ciudad (Noguera, 2003, pág. 69); casi simultáneamente, se expidió la Ley 46 de 1918, que ordena que el 2\% de los impuestos municipales se destinen a la construcción de una vivienda higiénica que se exigía como solución y mejora en la calidad de la vida para estas personas. (Cotrino Diaz, 2007, pág. 7)

Vivienda higiénica y la aplicación de los postulados de la arquitectura moderna

Ante la situación social que se vivía, comenzaron a construirse en Colombia viviendas higiénicas para los sectores obreros y campesinos, al entrar en vigor las premisas del movimiento moderno. (Orozco, 2018, pág. 37) Eran viviendas, que compartían un mismo patrón, donde la iluminación y la ventilación conformaban espacios limpios (Cotrino Diaz, 2007, pág. 7), dignos para quienes las habitaban.

Con la construcción de vivienda higiénica a partir del año 1918 (Orozco, 2018, pág. 36) se comienza a aplicar los postulados de la arquitectura moderna para la clase obrera, para lo cual se determinan normas mínimas. (Orozco, 2018, pág. 36) La vivienda mínima es la respuesta a las condiciones de pobreza, segregación social, urbanización desordenada y de desborde de los mecanismos formales de organización de la ciudad (Pelli, 2007, pág. 94). 
En 1932 surge el Instituto de Acción Social (IAS) (Leyva, 1996), que fomentaba la construcción de viviendas bajo postulados modernos europeos, con el diseño de espacios garantizadores de dignidad humana. Cada urbanización que construía el Instituto, mejoraba en calidad a la anterior.

En la década de los 20, las viviendas para la clase obrera se construyeron bajo los parámetros modernos de la Arquitectura Moderna (flexibilidad, progresividad e higiene, y delimitación de los espacios privados y sociales) (Leyva, 1996, pág. 20); en los años 20 a 30 comenzó la construcción de edificios usando un modelo repetitivo (Leyva, 1996, pág. 22) , lo que comenzó a consolidar las unidades vecinales como modelo urbanístico y como modelo de vivienda (Leyva, 1996, pág. 24).

En los años siguientes se fueron creando más entidades que implementaron programas en donde las personas de clase media también podían acceder a la vivienda mínima; fue así como el Instituto de Acción Social (IAS), en 1934, comenzó a beneficiar a sus trabajadores dando pie a la inclusión de esos estratos medios a tener vivienda. (Cotrino Diaz, 2007, pág. 9) El diseño de estos barrios se basó en el concepto de ciudad jardín (Cotrino Diaz, 2007, pág. 10) lo que de alguna manera permitía que los habitantes que venían del campo tuvieran una vivienda con áreas libres que evocaba su procedencia.

A partir de los años 40, se empiezan a crear entidades públicas y privadas para proveer vivienda a clases medias y bajas, entre estas el Banco Central Hipotecario (BCH) en 1936, la Caja de Vivienda Popular, en 1942 (surgida a raíz de la Junta de habitaciones para obreros (1919-1932) y el Instituto de Acción Social (1932-1942)), el Instituto de Crédito Territorial en 1942 y el Ministerio de Obras Públicas, a comienzos de los 60 .

\section{Vivienda de interés "social" hoy}

A fA finales del siglo XX se crea la Vivienda de interés social. El Decreto 2190 de 2009, Reglamentario de la Ley 9 de 1989, Ley 3 de 1991, Ley 388 de 1997 y Ley 1151 de 2007, dentro del acápite de definiciones establece que Vivienda de Interés Social (VIS) es aquella que reúne los elementos que aseguran su habitabilidad, estándares de calidad en diseño urbanístico, arquitectónico y de construcción cuyo valor máximo es de ciento treinta y cinco salarios mínimos legales mensuales vigentes $(135 \mathrm{smlm})$. 
Actualmente las políticas públicas de vivienda están orientadas a programas para construcción y mejora de vivienda, manejando en algunos casos el sistema de autoconstrucción. De esta manera se le da un papel importante a la comunidad en el proceso de la construcción de la casa, con el objeto de lograr un óptimo resultado gracias a la cooperación entre los roles de coordinación, financiación, empresas, ONGs y comunidad (Olaya, 2013, pág. 18).

El mayor número de proyectos de Vivienda de Interés Social y de interés prioritario se adelantan actualmente en Bogotá, en las localidades de Bosa y Ciudad Bolívar (Bogotá, 2018, pág. 16), dirigidas a familias víctimas del conflicto armado, a madres cabeza de familia, a población desbancarizada y pequeños ahorradores (Bogotá, 2018, pág. 65). Algunos proyectos en Bogotá utilizan un nuevo sistema de financiación que incluye procesos de captura de valor, cargas urbanísticas, alianzas con el sector privado, aportes del Distrito Capital para la financiación de vivienda, derechos de construcción y desarrollo, entre otros.

Vivienda popular de los años 50 vs. Vivienda de interés social actual. Una comparación.

A continuación se hace una comparación entre una vivienda unifamiliar del barrio Muzú en Bogotá, construido en 1949 por el Estado aplicando los preceptos de la arquitectura moderna y una vivienda unifamiliar del barrio el barrio Colinas de Zué en Tunja, proyecto de un constructor privado.

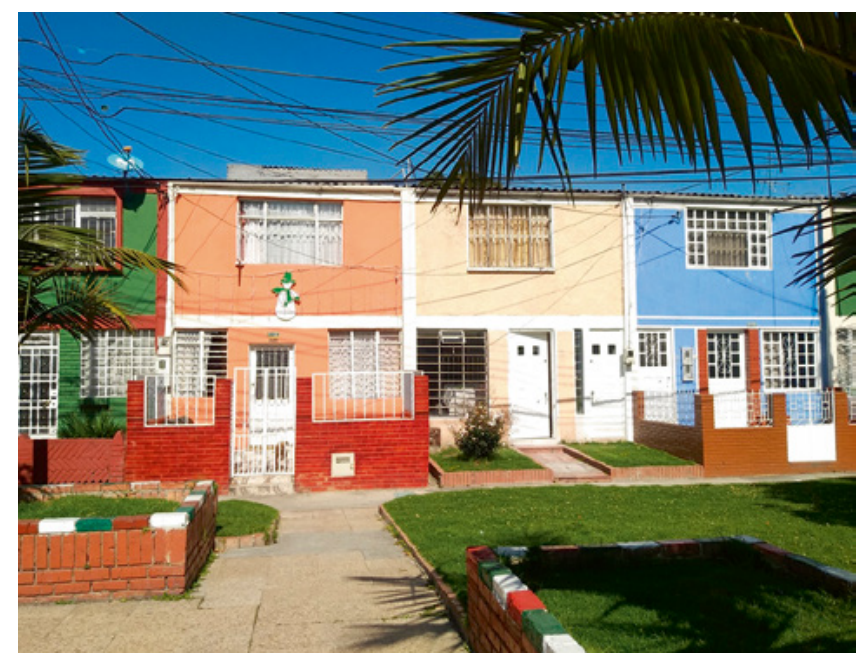

Figura 1. Fachada de casas barrio Muzú, Bogotá. Fuente: Ana María Alami Zambrano, 2019. 


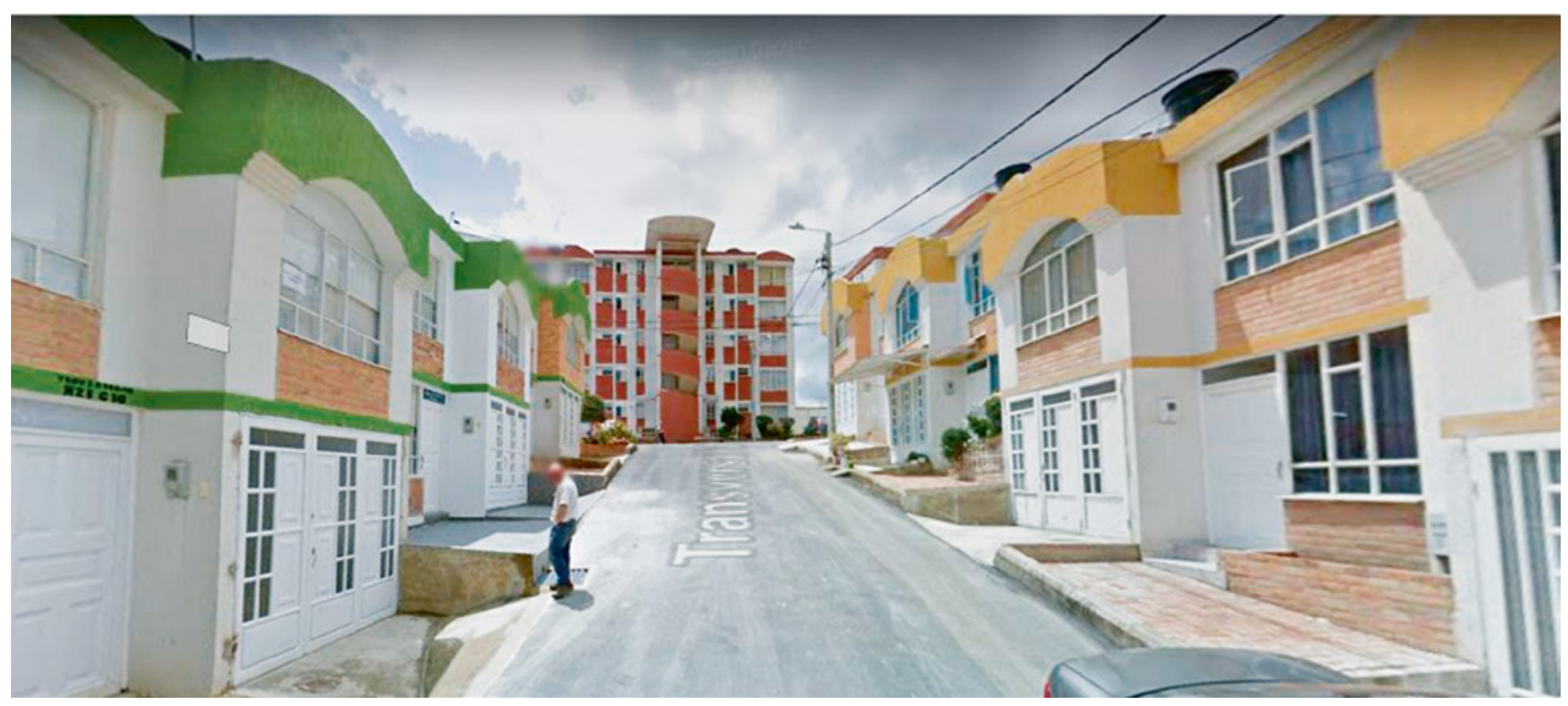

Figura 2. Fachada de casas barrio Colinas del Zué, Tunja. Fuente: Tomada de Googlemaps Feb. 2013.

El barrio Muzú, está ubicado en la ciudad de Bogotá; fue construido en 1949 por el Instituto de Crédito Territorial, diseñado por el departamento de diseño de la misma entidad.

Cuenta con conexiones urbanas eficientes, ya que sus calles y carreras corresponden a un trazado ordenado que les permite a sus habitantes conectarse al exterior con facilidad a vías principales que comunican con equipamientos importantes como: Iglesia Santa Isabel de Hungría, coliseo cubierto Santa Isabel de Hungría, colegio Santa Isabel de Hungría, colegio Distrital Andrés Bello, colegio Julio Garavito Armero, canchas de fútbol Muzú, unidad médica Santa Isabel de Hungría, estadio la alquería, CADE Muzú, colegio Fernando V de Aragón y el salón comunal Muzú. El siguiente esquema grafica lo anteriormente expresado. 


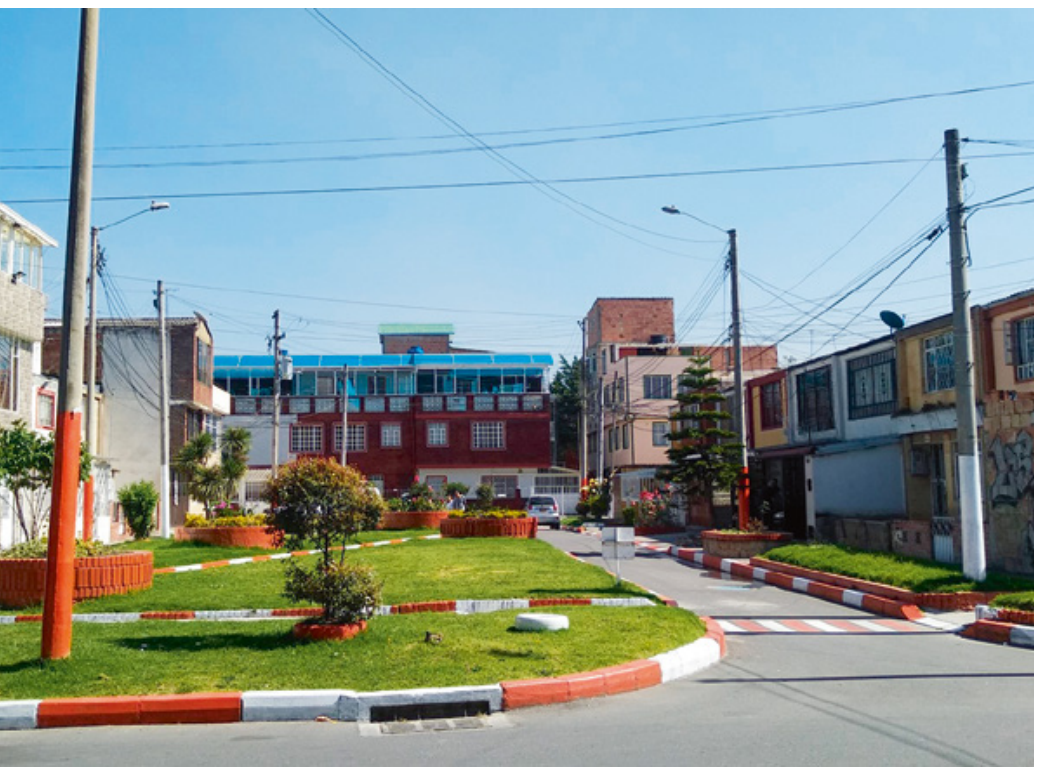

Figura 3. Análisis urbano. Fuente: Ana María Alami Zambrano, 2019.

Figura 4. Parque barrio Muzú, Bogotá. Fuente: Ana María Alami Zambrano, 2019.

La composición interna del barrio responde con un 30\% de su área dispuesto a espacio público, necesidad latente en complejos de vivienda popular de esta escala. En Muzú se encuentran 22 parques disponibles para los habitantes del sector, que sirve como espacios de ocio, recreación e interacción vecinal. Estos parques embellecen 
el entorno trasmitiéndoles a sus habitantes armonía, seguridad y aire limpio, casi un privilegio en Bogotá.

A continuación se ilustra una casa del barrio Muzú, es un ejemplo de la enorme influencia del modernismo en 1949; su diseño garantiza una vivienda digna, con espacios amplios, ventilados e iluminados, en pro del desarrollo para la vida interna familiar.

En la primera planta, cuentan las casas con un antejardín de $4 \mathrm{M}$ como zona de transición del exterior al interior. A la vivienda se accede por un único acceso. Inmediatamente hay una sala de $21 \mathrm{~m} 2$ y un punto fijo iluminado naturalmente. La cocina, contigua a la sala, tiene $21 \mathrm{~m} 2$, con iluminación natural y ventilación a través de un aislamiento posterior de $9 \mathrm{M}$, el cual se usa como patio de ropas y como área de recreación para la familia. La vivienda cuenta con un solo baño ubicado en el primer nivel, el cual sirve a áreas sociales y privadas. En la segunda planta se encuentran tres habitaciones con iluminación natural cenital y lateral; las tres comprenden un área total de $59 \mathrm{~m} 2$

La distribución de los espacios se observa en el siguiente esquema:

CASA BARRIO MUZÚ
BOGOTÁ - COLOMBIA

\section{ÁREA TOTAL: $205 \mathrm{~m} 2$}
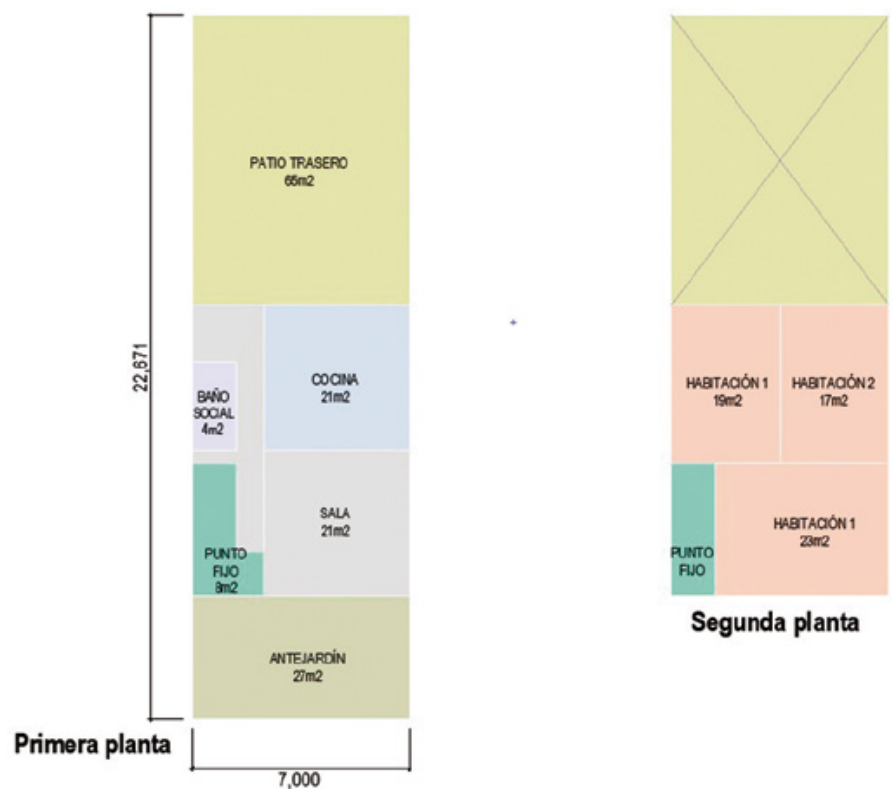

Figura 5. Esquema de distribución espacial de una casa del barrio Muzú. Fuente: Ana María Alami Zambrano, 2019. 
A continuación, se describe el barrio Colinas de Zué, proyecto de Vivienda de Interés Social, ubicado en la ciudad de Tunja, realizado por el ingeniero Ricardo Vargas, constructor privado.

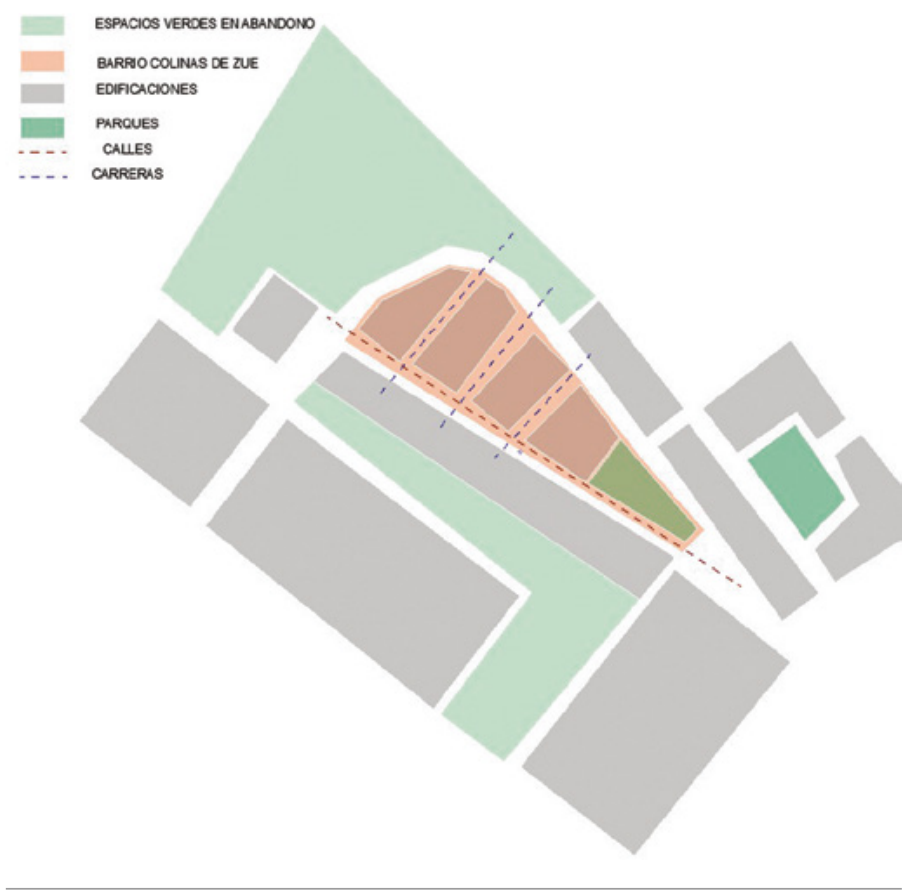

Figura 6. Plano urbano Colinas de Zué en Tunja. Fuente: Elaborado por Ana María Alami Zambrano, 2019.

Colinas de Zué es un ejemplo de la vivienda de interés social construida para familias de ingresos medios, que suplir la necesidad de vivienda, respondiendo con espacios con áreas mínimas, algunos con iluminación y ventilación, otros no. Si bien en el entorno hay equipamientos importantes como el colegio Andino de Tunja, Universidad de Boyacá, Clínica Medilaser, entre otros, el diseño urbano no plantea conexiones con estos y con los demás usos complementarios de la zona.

En la primera planta se encuentran dos accesos, uno para el peatón y otro para garaje con un área de $12 \mathrm{~m} 2$, antecedidos por un antejardín de 0,50 Mts, utilizado como espacio para algunas plantas, pues por su dimensión no es posible realizar 
en él ninguna actividad. $\mathrm{Al}$ ingresar a la vivienda se encuentra la sala de $23 \mathrm{~m} 2 \mathrm{con}$ óptima iluminación natural, seguido a esta, la cocina con $12 \mathrm{~m} 2$ ventilada e iluminada a través de un patio con $9 \mathrm{~m} 2$, el cual, dadas las condiciones del clima de Tunja, está cubierto con marquesina en primer piso, eliminando toda posibilidad de ventilación natural en la primera planta. En esta primera planta hay un baño de $2.25 \mathrm{~m} 2$ para las áreas sociales ubicado junto al punto fijo que conduce a la segunda planta, en la que hay tres habitaciones, 2 con baño privado y otro ubicado en la parte exterior de las habitaciones; 2 de los baños de esta planta carecen de ventilación e iluminación. En esta misma planta está el punto fijo que conduce al altillo de $18 \mathrm{~m} 2$, con baño privado ventilado e iluminado. El siguiente esquema ilustra lo anteriormente descrito:

CASA BARRIO COLINAS DE ZUE TUNJA - COLOMBIA

156

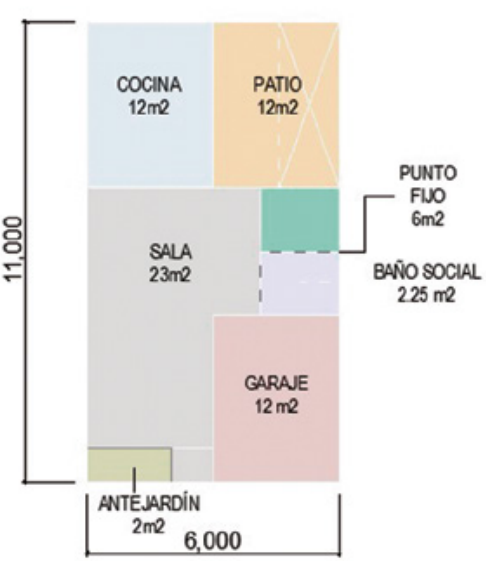

PISO 1
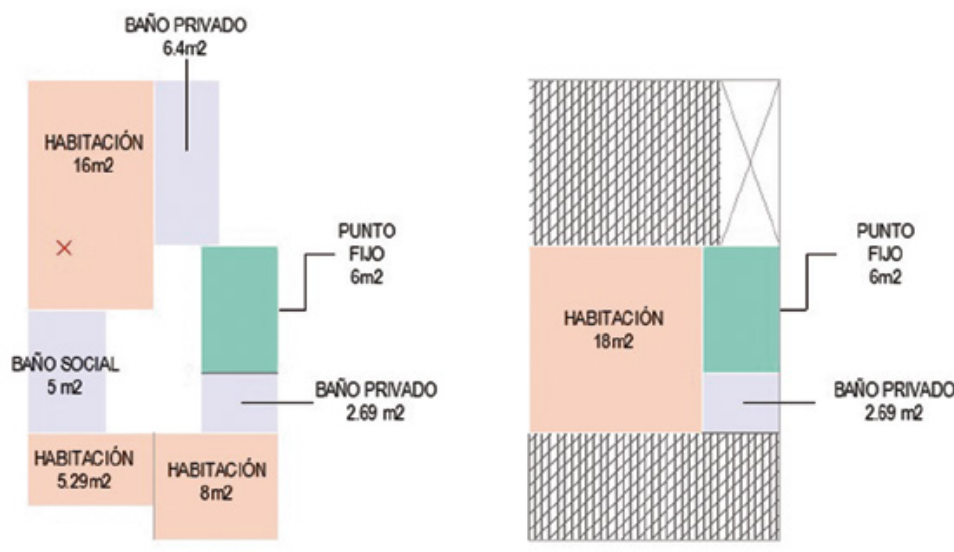

PISO 2
ÁREA TOTAL: $143 \mathrm{~m} 2$

Figura 7. Esquema de distribución espacial vivienda barrio Colinas de Zué. Fuente: Elaborado por Ana María Alami Zambrano, 2019. 
La siguiente tabla muestra una comparación entre las áreas de uno y otro barrio:

\begin{tabular}{|c|c|c|}
\hline ESPACIO & MUZU & $\begin{array}{c}\text { COLINAS } \\
\text { DE ZUÉ }\end{array}$ \\
\hline Jardín & $27 \mathrm{M} 2$ & $2 \mathrm{M} 2$ \\
\hline Sala & $21 \mathrm{M} 2$ & $23 \mathrm{M} 2$ \\
\hline Cocina & $21 \mathrm{M} 2$ & $12 \mathrm{M} 2$ \\
\hline Patio & $66 \mathrm{M} 2$ & $12 \mathrm{M} 2$ \\
\hline $\begin{array}{c}\text { Habitación (pro- } \\
\text { medio) }\end{array}$ & $20 \mathrm{M} 2$ & $14 \mathrm{M} 2$ \\
\hline $\begin{array}{c}\text { Baños } \\
\text { (Promedio) }\end{array}$ & $4 \mathrm{M} 2$ & $4 \mathrm{M} 2$ \\
\hline
\end{tabular}

\section{CONCLUSIONES}

Considerar la arquitectura moderna como patrimonio es una prioridad; su importante papel en la dignificación de la clase popular amerita destacarse, pues la construcción de vivienda obrera trajo consigo un nuevo estilo de vida en respuesta a las necesidades de vivienda y la solución a problemáticas higiénicas y sociales. Estas urbanizaciones contaban con un planeamiento urbanístico que les brindó conexiones fundamentales a servicios de equipamientos de salud, religiosos, educativos, comerciales y deportivos.

En la construcción de viviendas populares fueron tan importantes los espacios públicos. Los espacios verdes equilibraron el impacto de la densificación y se aprovecharon para el fortalecimiento de interacciones entre vecinos. Estos espacios también contribuyeron a la sensibilización de los habitantes por el medio ambiente. 
Las viviendas construidas en su momento para clase popular, hoy día, son valoradas en altos precios desde $\$ 300^{\prime} 000.000$ en adelante. Estos sectores se han convertido en barrios en los que actualmente es un privilegio vivir. Las Viviendas de Interés Social actuales no son comparables con lo que fueron las viviendas populares; las casas y apartamentos VIS de hoy está diseñados con áreas mínimas, que ignoran la dignidad de sus ocupantes ya que la iluminación, la ventilación y la amplitud han pasado a un segundo plano. Por otro lado, las conexiones a equipamientos son deficientes: calles sin pavimentar y lejos de espacios públicos y a equipamientos de uso comercial, salud, educación y de recreación deportiva.

La dignidad humana en la vivienda debe ser un objetivo desde el poder, pues allí está el presupuesto para planificar y aplicar las soluciones; reestablecer normativas constructivas y espaciales de las Viviendas de Interés Social, sería el primer paso. Encomendar estos proyectos a arquitectos con largos recorridos profesionales especialistas en la materia, debería ser la manera de selección de profesionales que desarrollen proyectos de este tipo; de esta manera se garantiza la excelencia en proyectos para la comunidad desde su planificación, construcción y vivencia pues los habitantes hoy, de clase baja y media, con mínimos recursos para la adquisición de vivienda digna, sueñan con un espacio asequible e íntegro. 


\section{REFERENCIAS BIBLIOGRÁFICAS}

Arango, S. (1993). Historia de la arquitectura en Colombia. Bogotá. Bogotá: Universidad Nacional de Colombia.

Bogotá, D. (2018). Informe de resultados, plan distrital de desarrollo, Bogotá mejor para todos. Bogotá.

Cotrino Diaz, Y. (2007). La vivienda obrera ¿Un elemento apaciguador o modernizador? La intervención del Estado en Bogotá 1918-1942. Revista de Arquitectura Universidad Católica.

Leyva, C. C. (1996). Estado, ciudad y vivienda: urbanismo y arquitectura de la vivienda estatal en Colombia 1918-1990. Puntos Suspensivos Editores.

Mayor Mora, A. (2002). El nacimiento de la industria colombiana. Un parto de hierro, hidráulica y trabajo femenino e infantil. Credencial historia No. 151.

Noguera, C. (2003). Medicina y Política: discurso médico y prácticas higiénicas durante la primera mitad del siglo $x x$ en Colombia.

Olaya, A. B. (2013). “Vivienda de Interés Social de calidad en Colombia: hacia una solución integral." .

Orozco, J. C. (2018). La vivienda mínima: una revisión del desarrollo del concepto en Colombia. Procesos Urbanos.

Pelli, V. S. (2007). Habitar, participar, pertenecer acceder a la vivienda. 